\title{
SARS-CoV-2 infection prevalence in healthcare workers and administrative and support staff: The first-wave experience at three academic hospitals in the Tshwane district of Gauteng Province, South Africa
}

\author{
N Mdzinwa, ${ }^{1} \mathrm{MB} \mathrm{ChB}, \mathrm{DA}(\mathrm{SA}) ; \mathbf{M}$ Voigt ${ }^{2} \mathrm{MB} \mathrm{ChB}, \mathrm{DA}(\mathrm{SA}), \mathrm{MMed}$ (Anaesth), FCA (SA); C Janse van Rensburg, ${ }^{3} \mathrm{MSc}$; \\ F Paruk, ${ }^{4} \mathrm{MB}$ ChB, FCOG (SA), Cert Crit Care (SA), PhD \\ ${ }^{1}$ Department of Anaesthesiology, Steve Biko Academic Hospital and School of Medicine, Faculty of Health Sciences, University of Pretoria,
South Africa
${ }^{2}$ Department of Anaesthesiology, Kalafong Provincial Tertiary Hospital and School of Medicine, Faculty of Health Sciences, University of Pretoria,
South Africa
${ }^{3}$ Biostatistics Unit, South African Medical Research Council, Pretoria, South Africa
${ }^{4}$ Department of Critical Care, Steve Biko Academic Hospital and School of Medicine, Faculty of Health Sciences, University of Pretoria, South Africa
}

Corresponding author: N Mdzinwa (nasiphiM17@gmail.com)

\begin{abstract}
Background. The availability of well and functional healthcare workers (HCWs) and support staff is pivotal to a country's ability to manage the COVID-19 pandemic effectively. While HCWs have been identified as being at increased risk for acquisition of SARS-CoV-2 infection, there is a paucity of data pertaining to South African (SA) HCW-related infection rates. Global and provincial disparities in these numbers necessitate local data in order to mitigate risks.

Objectives. To ascertain the overall SARS-CoV-2 infection rates and outcomes among all hospital staff at three hospitals in the Tshwane district of Gauteng Province, SA, and further determine associations with the development of severe COVID-19 disease.

Methods. This retrospective audit was conducted across three academic hospitals in the Tshwane district for the period 1 June - 31 August 2020. Deidentified data from occupational health and safety departments at each hospital were used to calculate infection rates. A more detailed analysis at one of the three hospitals included evaluation of demographics, work description, possible source of SARS-CoV-2 exposure (community or hospital), comorbidities and outcomes.

Results. The period prevalence of SARS-CoV-2 infections ranged from $6.1 \%$ to $15.4 \%$ between the three hospitals, with the average period prevalence being $11.1 \%$. The highest incidence of SARS-CoV-2 infections was observed among administrative staff ( 2.8 cases per 1000 staff days), followed by nursing staff ( 2.7 cases per 1000 staff days). Medical doctors had the lowest incidence of 1.1 cases per 1000 staff days. SARS-CoV-2 infections were categorised as either possibly community or possibly healthcare facility acquired for $26.6 \%$ and $73.4 \%$ of the infections, respectively. The administrative group had the highest proportion of possible community-acquired infections (41.8\%), while doctors had the lowest (6.1\%). The mean age of individuals with mild and severe disease was 41 years and 46.1 years, respectively $(p=0.004)$. The presence of comorbidities was significantly associated with severity of disease $(p=0.002)$.

Conclusions. This study highlights that hospital staff, including administrative staff, are clearly at high risk for acquisition of SARS-CoV-2 infection during a surge.
\end{abstract}

S Afr Med J 2021;111(11):1092-1097. https://doi.org/10.7196/SAMJ.2021.v111i11.15938

\begin{abstract}
A physically and mentally well healthcare workforce is vital to a country's ability to manage patients effectively in the face of the SARS-CoV-2 pandemic. ${ }^{[1]}$ South African (SA) healthcare workers (HCWs) have played a relentless and crucial role in the fight against SARS-CoV-2 infections, and continue doing so. HCWs have had to rapidly adapt practices and face high risks of burnout, ${ }^{[2]}$ stigmatisation and physical exhaustion, as well as witness the loss of their patients, friends and family members. Furthermore, hospital administrative staff and support staff have also provided essential services to ensure the efficiency of healthcare services. Against this backdrop, many HCWs and essential support staff have acquired SARS-CoV-2 infections and a substantial number have died - as of 4 August 2020, there had been 27360 infections and 443 deaths among SA HCWs. ${ }^{[3]}$
\end{abstract}

The majority of the HCW infections (78\%) were in the public sector, while the majority of HCW deaths were in the private health sector $(54 \%) .{ }^{[3]}$ The vast majority of HCW-related infections have occurred among nurses. Infection and mortality rates have differed across provinces, but the reported national HCW infection rate of $5 \%$ in August 2020 was well below the $10 \%$ global average. ${ }^{[3]}$ Global data indicate that $\mathrm{HCW}$ infection rates range from $4 \%$ to $20 \%{ }^{[4-9]}$

Variations in reported HCW infection rates can be attributed to a multitude of reasons, including human resource shortages, personal protection equipment (PPE) quality concerns or shortages thereof, duration of exposure, inadequate hand hygiene, poor compliance with infection prevention and control (IPC) policies, aerosolisation risks, or the type of service provided by the discipline. From an SA perspective, there is a paucity of data pertaining to infection rates among hospital staff. The global and national variations in risk of infection acquisition necessitate gathering of local data in order to identify gaps and to mitigate risk where necessary. 


\section{Objectives}

To ascertain the overall SARS-CoV-2 infection rates and outcomes among all hospital staff (HCWs, administrative staff and support staff) at three academic hospitals in the Tshwane district of Gauteng Province (GP), SA. Additionally, we sought to determine associations, if any, with the development of severe disease.

\section{Methods}

This retrospective audit was conducted at Steve Biko Academic Hospital (SBAH), Dr George Mukhari Academic Hospital (DGMAH) and Kalafong Provincial Tertiary Hospital (KPTH) for the period 1 June - 31 August 2020. The human resources department of each hospital provided their staff register. Deidentified records from the occupational health and safety department (OHSD) of each hospital were accessed to determine the number of staff positive for SARSCoV-2 infection for the study period.

The OHSD records for the SBAH cohort were additionally utilised to capture staff demographics, work description, likely source of SARS-CoV-2 exposure (community or hospital), comorbidities and outcomes. While we initially intended to do this for all hospitals, it was not possible for DGMAH and KPTH owing to a high proportion of missing variables.

Ethical clearance was obtained from the University of Pretoria Research Ethics Committee (ref. no. 325/2020).

The SBAH cohort was allocated to one of the following categories:

Administrative staff. Office-based staff and administrative staff based at hospital entry points to the wards or theatre complexes. For administrative staff, precautions are in place to avoid direct patient contact.

Allied healthcare professionals. All healthcare professionals other than nurses and medical practitioners, including pharmacists, dieticians, physiotherapists, clinical technologists, radiographers and occupational therapists.

Medical doctors. All medical practitioners, including trainees, interns and medical hospital managers.

Nursing staff. All nursing staff, including nursing managers.

Support staff. Non-HCWs who play a role in ensuring the efficiency of hospital services, including cleaners, porters and foodcatering staff.

An infection was ascribed to being possibly community acquired or possibly healthcare facility acquired based on documentation of a known contact in the community or healthcare facility, respectively. Severity of SARS-CoV-2 infection was defined as mild (not requiring hospitalisation), moderate (necessitating hospital admission), or severe (ICU admission or death). For the purposes of analysis, we combined the moderate and severe infections into the severe category.

The hospital staff database provided by the respective human resources departments was used to categorise the staff work description and to calculate infection rates for each hospital. The Coronavirus COVID-19 (2019-nCoV) Data Repository for South Africa was used to access national and provincial statistics to permit infection rate comparisons with available Gauteng provincial and national data for the general population.

\section{Statistical analysis}

Frequencies and proportions were used to describe categorical variables. Continuous variables were described using means and standard deviations (SDs). The $\chi^{2}$ test was used to determine crude associations between categorical variables, including severity of SARS$\mathrm{CoV}-2$ infections. In order to estimate incidence, data were aggregated at level of staff category (administrative, allied healthcare professionals, medical doctors, nurses, support) by month of observation (June, July, August). The number of staff in each of the categories was assumed to be the same for each month. Staff days per month (exposure) for each staff category was calculated as the number of staff in the category $x$ the number of days in the month ( 30 for June, 31 for July and August) $\times 5$ days (assumed number of days per week). For doctors, the assumed number of days per week was 6 . Poisson regression analysis was used to model the number of SARS-CoV-2 cases for a staff category in a month, with staff days/1 000 as exposure. Crude unadjusted incidence is reported as the predicted number of cases per 1000 staff days with associated $95 \%$ confidence intervals (CIs). To estimate incidence for months over hospitals, and vice versa, models were fitted with an intercept and main effect for month and hospital, respectively. A model with main effects for month and hospital, as well as an interaction term, was used to estimate incidence at each month-hospital combination. A similar approach was used to estimate incidence for staff groups across months for SBAH. Model-based inference was done in order to compare the incidence between SBAH and the other hospitals. The incidence for doctors was compared with the average incidence for the other four staff categories, also using the model estimates. Observed case and death data for SA and GP were retrieved for June - August 2020 from the Coronavirus COVID-19 (2019-nCoV) Data Repository for South Africa. Mid-year population estimates for 2020 for SA and GP were retrieved from the relevant report by Statistics South Africa. ${ }^{[10]}$ All analysis was conducted using Stata 16 (StataCorp, USA). Statistical significance was set at $5 \%$.

\section{Results}

The period prevalence of SARS-CoV-2 infections ranged from $6.1 \%$ (95\% CI $5.1-7.2)$ to $15.4 \%$ (95\% CI 14.2 - 16.6) in the three hospitals (Table 1), with an overall average of $11.1 \%$ (95\% CI 10.5 - 11.7). SBAH demonstrated the highest percentage of infections.

During the same period, 594089 new cases were observed in SA, of which 205875 were recorded in GP. This translates to $0.1 \%$ and $1.3 \%$ of people being infected in SA and GP, assuming 2020 midyear population estimates of 59.62 million and 15.5 million people, respectively. ${ }^{[10,11]}$

\section{Incidence of SARS-CoV-2 infections}

The study hospitals, GP and SA exhibited a similar trajectory in terms of the rise and fall of infections during the study period, with

Table 1. Period prevalence of SARS-CoV-2 infections, June - August 2020

\begin{tabular}{lllll}
\hline Indicator & SBAH & DGMAH & KPTC & \multicolumn{1}{c}{ Total } \\
\hline Total staff employed, $n$ & 3682 & 4800 & 2092 & 10574 \\
Staff days, $n$ & 247913.7 & 315428.57 & 137474.4 & 700816.6 \\
SARS-CoV-2 infections, $n$ & 567 & 482 & 127 & 1176 \\
Period prevalence, \% (95\% CI) & $15.4(14.2-16.6)$ & $10.0(9.2-10.9)$ & $6.1(5.1-7.2)$ & $11.1(10.5-11.7)$ \\
SBAH = Steve Biko Academic Hospital; DGMAH $=$ Dr George Mukhari Academic Hospital; KPTH = Kalafong Provincial Tertiary Hospital; CI = confidence interval.
\end{tabular}


infections rising sharply in June, peaking in late July and declining towards the end of August (Fig. 1).

The overall incidence of infections among all hospital staff was 1.7 (95\% CI 1.6 - 1.8) per 1000 staff days (Table 2). SBAH demonstrated a significantly higher incidence of 2.3 (95\% CI 2.1 - 2.5) compared with DGMAH, which had an incidence of 1.5 (95\% CI 1.4 - 1.7) $(p<0.001)$ and KTPH, which had an incidence of 0.9 (95\% CI 0.7 1.1) $(p<0.001)$.

\section{Severity of SARS-CoV-2 infections}

Hospital admission was necessary for $7.8 \%$ and $2.7 \%$ of SARS-CoV-2 infections for SBAH and DGMAH hospital staff, respectively. The observed case fatality rate was $0.8 \%$ for both hospitals. There were 4 deaths in the SBAH cohort and 2 in the DGMAH cohort.

A high proportion of missing variables in the records at DGMAH and KPTH precluded their inclusion for any further analysis, so the results that follow are restricted to SBAH.

\section{Steve Biko Academic Hospital}

SBAH had 3682 staff members during the study period, of whom 567 acquired SARSCoV-2 infection. The highest incidence of SARS-CoV2 infections (Table 3 and Fig. 2) was observed among administrative staff, who experienced 2.8 (95\% CI 2.2 3.4) cases per 1000 staff days, followed by nursing staff with 2.7 (95\% CI 2.3 3.0). Medical doctors displayed the lowest incidence, 1.1 (95\% CI 0.8 - 1.4), which is significantly lower than the averaged incidence across the other staff categories $(p<0.001)$.

The mean (SD) age of the 567 SBAH staff members who acquired SARS-CoV-2 infection was 41.3 (10.3) years, and the majority of infections were among females (82.7\%). Nursing staff comprised the largest staff group developing an infection (47.6\%), followed by support staff (22.1\%), administrative staff (15.3\%), allied healthcare professionals $(7.8 \%)$ and medical doctors (7.2\%). Furthermore, $26.6 \%$ and $73.4 \%$ of SARS-CoV-2 infections were categorised to be probably community- or healthcare facility-acquired infections, respectively.

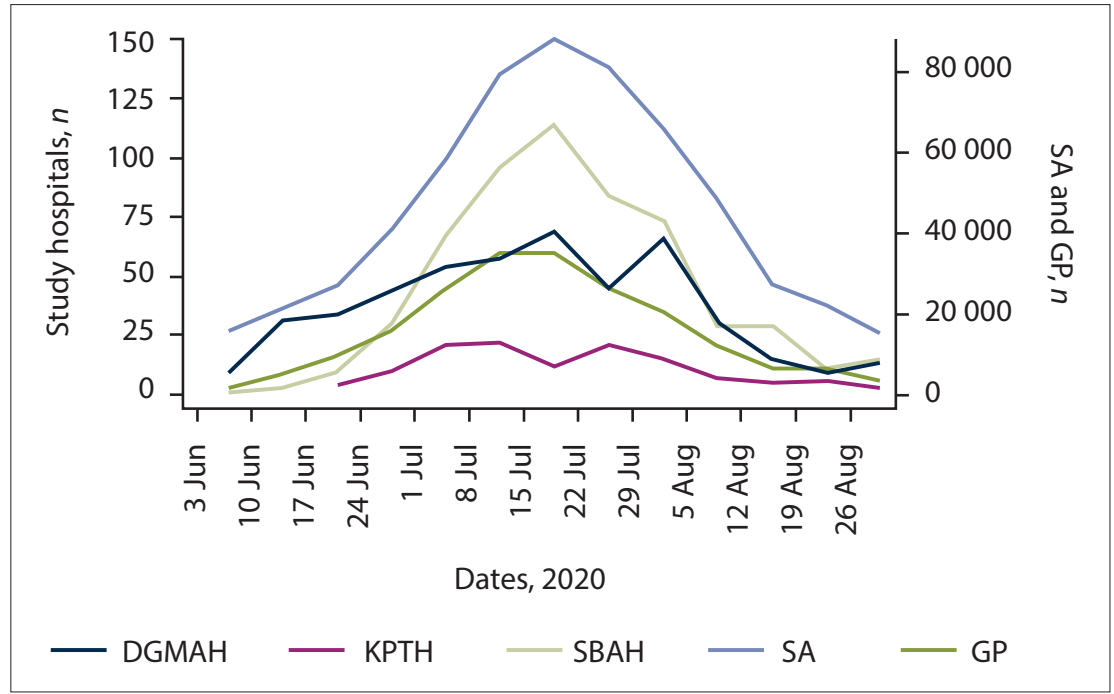

Fig. 1. Weekly number of new cases for the study hospitals, GP and SA. (GP = Gauteng Province; $S A=$ South Africa; DGMAH = Dr George Mukhari Academic Hospital; KPTH = Kalafong Provincial Tertiary Hospital; SBAH = Steve Biko Academic Hospital.)
The breakdown of community v. hospital acquisition is shown in Table 4

Severity of disease could be categorised for 498 of the 567 SARS-CoV-2 infections (87.8\%) (Table 5). Support staff experienced the highest proportion of severe cases $(10.6 \%)$ and medical doctors the lowest (8.1\%); however, this difference was not significantly different for the various staff categories $(p=0.745)$. The mean (SD) age of those who had mild and severe disease was 41.0 (10.3) years and 46.1 (10.3) years, respectively, and was found to differ significantly $(p=0.004)$. The presence of comorbidities was significantly associated with severity of disease $(p=0.002)$.

\section{Discussion}

The study demonstrated an overall period prevalence of $11.1 \%$ (95\% CI 10.5 - 11.7) of SARS-CoV-2 infections among all hospital staff, which is significantly higher than the $1.3 \%$ prevalence in the general population of GP for the same period. ${ }^{[10,11]}$ In the Tshwane region, the earlier preparedness of SBAH initially resulted in acceptance of SARSCoV-2 patient transfers from Thembisa Hospital, DGMAH and KPTH during the study period. Additionally, SBAH combined its workforce with Tshwane District Hospital $(\mathrm{TDH})$ to create the SBAH-TDH complex to manage SARS-CoV-2 patients. A selfscreening application was developed at SBAH and was available to all staff members. Staff who did not have access to this app were alternatively screened at the hospital entrances by completing a paper checklist. Infrared temperature measurements were taken on all staff members before they entered the hospital. Strict IPC practices were in place, with sanitisers available at the hospital entrances and in all corridors. Visual reminders were strategically placed at multiple points around the hospital to encourage social distancing, hand hygiene and wearing of face masks. Markings on the floor at clinics, pharmacies and other areas where queuing was anticipated served as reminders to ensure social distancing.

Table 2. Incidence (per 1000 staff days) of SARS-CoV-2 infections, June - August 2020

\begin{tabular}{|c|c|c|c|c|c|c|c|c|}
\hline & \multicolumn{2}{|r|}{ June } & \multicolumn{2}{|r|}{ July } & \multicolumn{2}{|r|}{ August } & \multicolumn{2}{|r|}{ Total } \\
\hline & $n$ & $95 \%$ CI & $n$ & $95 \%$ CI & $n$ & 95\% CI & $n$ & $95 \%$ CI \\
\hline DGMAH & 138 & $0.8(0.6-0.9)$ & 263 & $2.5(2.2-2.8)$ & 81 & $1.3(1.1-1.6)$ & 482 & $1.5(1.4-1.7)$ \\
\hline SBAH & 68 & $1.1(0.8-1.3)$ & 411 & $4.9(4.4-5.4)$ & 88 & $0.8(0.6-1.0)$ & 567 & $2.3(2.1-2.5)$ \\
\hline КРТН & 23 & $0.5(0.3-0.7)$ & 82 & $1.5(1.2-1.9)$ & 22 & $0.8(0.5-1.0)$ & 127 & $0.9(0.8-1.1)$ \\
\hline Total & 229 & $0.8(0.7-0.9)$ & 756 & $3.1(2.9-3.4)$ & 191 & $1.1(0.9-1.2)$ & 1176 & $1.7(1.6-1.8)$ \\
\hline
\end{tabular}

$\mathrm{CI}=$ confidence interval; DGMAH = Dr George Mukhari Academic Hospital; SBAH = Steve Biko Academic Hospital; KPTH = Kalafong Provincial Tertiary Hospital 


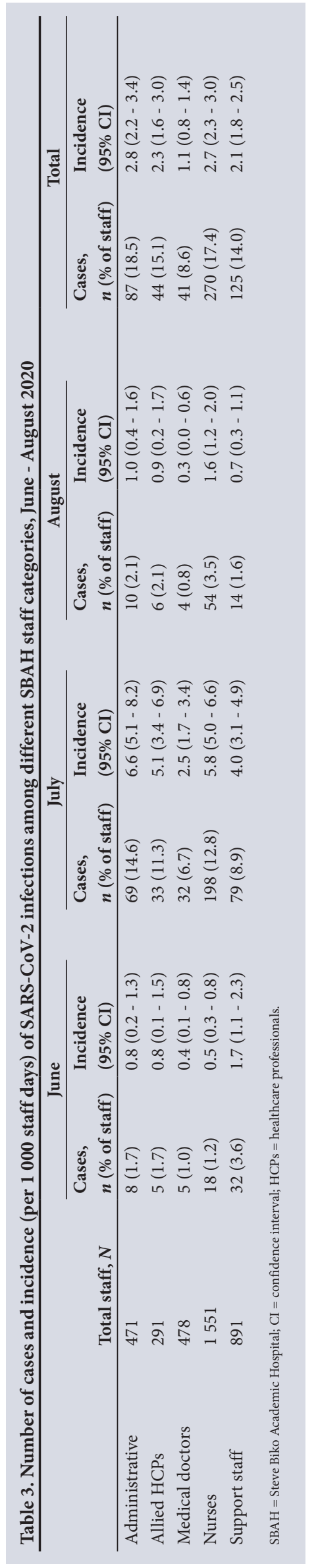

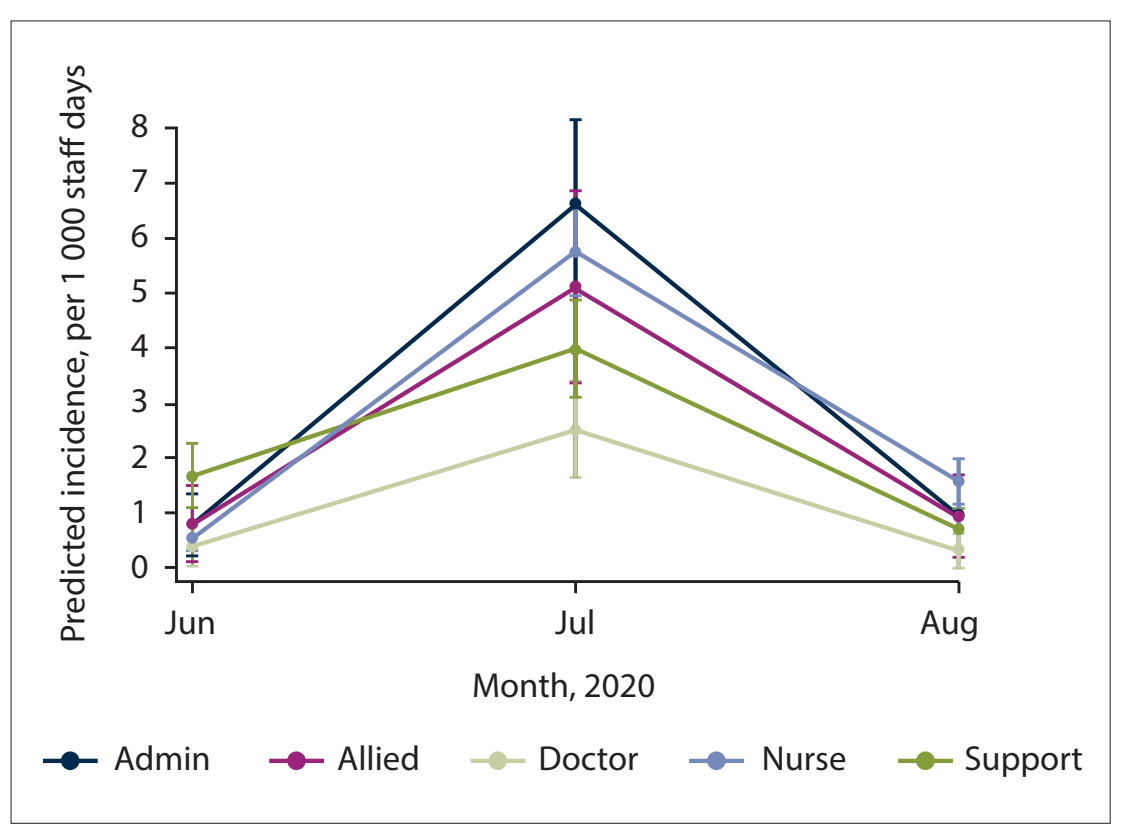

Fig. 2. Incidence of SARS-CoV-2 infections among the SBAH staff categories. ( $S B A H=$ Steve Biko Academic Hospital.)

Among hospital staff at SBAH, the period prevalence of $15.3 \%$ (95\% CI 13.8 - 16.8) may be attributed to various factors, including the sudden increase in workload, the need for staff to work in 'neo-wards', and crossdiscipline exposure to SARS-CoV-2 wards. The cross-discipline assistance by HCWs during the first-wave surge period precluded evaluation of duration and level of exposure per discipline, as well as discipline-related risks. As expected, the three study hospitals, GP and SA exhibited a similar trajectory for the incidence of new cases, in that there was a sharp rise in June, with a peak in late July and a subsequent decline towards the end of August. ${ }^{[10]}$ This parallel in the community and HCW trajectories is to be expected, as the number of infections in the community influences exposure of HCWs to the infection.

The high incidence of infections observed among the hospital staff is not unexpected, as previous studies have found HCWs to be at increased risk of acquiring SARS-CoV-2 infection. ${ }^{[4]}$ Our study is unique in that it included all hospital staff. ${ }^{[8]}$ We believe that it is important to ascertain infection rates even for essential workers with non-direct exposure to patients, such as administrative staff, who need to leave their homes during lockdown periods, travel to work, and then enter their workplace through a common hospital entrance. Most studies evaluating infection rates have either focused on staff who have the potential for direct or indirect exposure to patients or infectious materials, or not specified the level of exposure. ${ }^{[4]}$ An observational cohort study in the UK and in the USA of the general community, which included front-line HCWs, concluded that front-line HCWs were at increased risk for reporting a positive SARS-CoV-2 test. ${ }^{[4]}$ Furthermore, a systematic review and meta-analysis by Gómez-Ochoa et al. ${ }^{[9]}$ which included 97 studies, found the overall prevalence of SARS-CoV-2 infection to be $11 \%$ (95\% CI 7 - 15) among HCWs. ${ }^{[9]}$ Similarly, a study from Qatar reported a $10.6 \%$ positive test rate in a cohort of 16912 HCWs ${ }^{[6]}$ Ideally, a true estimate of hospital staff infection rates needs to consider and account for asymptomatic infections. Routine surveillance of hospital staff or HCWs for asymptomatic infections is not standard practice. ${ }^{[12]}$ Zou et al. ${ }^{[13]}$ demonstrated similar viral loads in symptomatic patients compared with asymptomatic individuals, highlighting the potential for transmission. While there has been a call for surveillance testing of HCWs to curtail intrahospital spread of SARS-CoV-2, its cost-effectiveness is unknown, and it highlights the importance of exploring screening tools. ${ }^{[14,15]}$

Our findings suggest that the majority of infections were likely to have been hospital acquired. However, even for infections thought to be acquired in the community, the contact is often not identified. Furthermore, it is difficult to control for unknown confounders, including asymptomatic infections among staff and patients, and in the community. The fact that the infection incidence was 2.8 (95\% CI $2.2-3.4)$ and 2.7 (95\% CI 2.3 - 3.0) cases per 1000 staff days for administrative and nursing staff, respectively, with the proportion of possible 
community-acquired infections being $41.8 \%$ and $17.2 \%$, illustrates this point. A study by Zaina et al. ${ }^{[16]}$ on HCWs found the highest percentage of infections to be among support and administrative staff at $5.6 \%$; however, $43.1 \%$ of these infections were community

\begin{tabular}{|c|c|c|c|}
\hline Staff category & Community & Hospital & Total \\
\hline \multicolumn{4}{|l|}{ Administrative } \\
\hline$n$ & 33 & 46 & 79 \\
\hline Row \% & 41.8 & 58.2 & 100 \\
\hline Col. \% & 25.2 & 12.7 & 16.02 \\
\hline \multicolumn{4}{|l|}{ Allied HCPs } \\
\hline$n$ & 11 & 30 & 41 \\
\hline Row \% & 26.8 & 73.2 & 100 \\
\hline Col. \% & 8.4 & 8.3 & 8.32 \\
\hline \multicolumn{4}{|l|}{ Doctors } \\
\hline$n$ & 8 & 26 & 34 \\
\hline Row \% & 23.5 & 76.5 & 100 \\
\hline Col. \% & 6.1 & 7.2 & 6.9 \\
\hline \multicolumn{4}{|l|}{ Nurses } \\
\hline$n$ & 39 & 188 & 227 \\
\hline Row \% & 17.2 & 82.8 & 100 \\
\hline Col. \% & 29.8 & 51.9 & 46.0 \\
\hline \multicolumn{4}{|l|}{ Support } \\
\hline$n$ & 40 & 72 & 112 \\
\hline Row \% & 35.7 & 64.3 & 100 \\
\hline Col. \% & 30.5 & 19.9 & 22.7 \\
\hline \multicolumn{4}{|l|}{ Total } \\
\hline$n$ & 131 & 362 & 493 \\
\hline Row \% & 26.6 & 73.4 & 100 \\
\hline Col. \% & 100 & 100 & 100 \\
\hline
\end{tabular}

acquired. HCW-to-HCW spread is thought to be more common than spread between HCW and patient; ${ }^{[7]}$ this may be attributed to the use of shared spaces, including tearooms and sleeping spaces, leading to poor compliance with social distancing protocols. ${ }^{[16]}$ In a report from Trieste, North-East Italy, cluster outbreaks were identified among HCWs, suggesting transmission among staff members rather than between patients and staff members. ${ }^{[7]}$

At the peak of the infections in July, the administrative group demonstrated the highest percentage of infections, probably because of community-acquired rates that were higher than the estimated rate. ${ }^{[16]}$ Administrative staff are mainly office based and work with minimal exposure to patients. Infections in this category probably tend to reflect individual IPC practices. Even if infections are community acquired, their high acquisition rate during lockdown restrictions suggests that essential workers are at higher risk of acquisition than the general community. The rate of infections among nurses was higher than that among doctors. This finding is in keeping with previous studies, which have attributed it as being due to longer patient contact time. ${ }^{[9]}$ The issue of shift working, communal tearooms and shared spaces also affects nurses more than doctors.

Hospital support staff are often in direct contact with the patients' environment, such as eating utensils and contaminated linen, despite there being no direct contact with patients. During the first month, the support staff category demonstrated the highest number of infections. This finding may be attributed to lack of familiarity with PPE, or the time required for the IPC learning curve at the start of the first wave. Doctors, nurses and allied healthcare professionals were already familiar with IPC, and mandatory comprehensive SARS-CoV-2related PPE training programmes would have further reinforced IPC strategies for them. Once cluster outbreaks were identified, renewed PPE training programmes were reinforced at SBAH.

In keeping with previous studies, we also found that age was associated with disease severity. ${ }^{[17,18]}$ The mean (SD) age in the mild group was 41.0 (10.3) years and that in the severe group 46.1 (10.3) years.

Table 5. Severity of cases according to various factors for the SBAH cohort and tests of associations with severity, June - August 2020

\begin{tabular}{|c|c|c|c|c|c|c|}
\hline & $\begin{array}{l}\text { Outpatient, } \\
n(\%)\end{array}$ & $\begin{array}{l}\text { Hospital } \\
\text { admission, } n(\%)\end{array}$ & $\begin{array}{l}\text { ICU, } \\
n(\%)\end{array}$ & $\begin{array}{l}\text { Death, } \\
n(\%)\end{array}$ & $\begin{array}{l}\text { Total, } \\
n(\%)\end{array}$ & $p$-value \\
\hline Gender & & & & & & 0.942 \\
\hline Female & 381 (91.8) & $25(6.0)$ & $5(1.2)$ & $4(1.0)$ & $415(100)$ & \\
\hline Male & $76(91.6)$ & $7(8.4)$ & 0 & 0 & $83(100)$ & \\
\hline Total & 457 (91.8) & $32(6.4)$ & $5(1.0)$ & $4(0.8)$ & $498(100)$ & \\
\hline Staff category & & & & & & 0.745 \\
\hline Admin & 75 (94.9) & $4(5.1)$ & 0 & 0 & $79(100)$ & \\
\hline Allied HCPs & 38 (92.7) & $3(7.3)$ & 0 & 0 & $41(100)$ & \\
\hline Doctors & 34 (91.9) & $2(5.4)$ & $1(2.7)$ & 0 & $37(100)$ & \\
\hline Nurses & 209 (91.7) & $13(5.7)$ & $2(0.9)$ & $4(1.8)$ & $228(100)$ & \\
\hline Support & $101(89.4)$ & $10(8.8)$ & $2(1.8)$ & 0 & $113(100)$ & \\
\hline Total & 457 (91.6) & $32(6.4)$ & $5(1.0)$ & $4(0.8)$ & $498(100)$ & \\
\hline Contact setting & & & & & & 0.006 \\
\hline Community & $113(86.3)$ & $14(10.7)$ & $2(1.5)$ & $2(1.5)$ & $131(100)$ & \\
\hline Hospital & 340 (93.9) & $18(5.0)$ & $3(0.8)$ & $1(0.3)$ & $362(100)$ & \\
\hline Total & 453 (91.9) & $32(6.5)$ & $5(1.1)$ & $3(0.6)$ & $493(100)$ & \\
\hline Risk factors & & & & & & 0.002 \\
\hline Comorbidities & $187(87.8)$ & $20(9.4)$ & $3(1.4)$ & $3(1.4)$ & $213(100)$ & \\
\hline None & $268(95.4)$ & $12(4.3)$ & $1(0.4)$ & 0 & $281(100)$ & \\
\hline Total & 455 (91.9) & $32(6.5)$ & $4(0.8)$ & $3(0.6)$ & $494(100)$ & \\
\hline
\end{tabular}


The significant association between the presence of comorbidities and disease severity at SBAH is supported in previous studies conducted in the general population. ${ }^{[18,19]}$ These comorbidities include cardiovascular disease, diabetes mellitus, chronic kidney disease, chronic respiratory disease, hypertension and malignancy. Obesity and smoking have also been reported to be associated with an increased risk of severe disease. ${ }^{[18,19]}$ SBAH recorded a $0.8 \%$ staff fatality rate for SARS-CoV-2 infections during the study period. This figure is far lower than the case fatality rates of $2.3 \%$ and $1.7 \%$ for the same period for SA and GP, respectively, but the number of severe staff cases was too low to permit meaningful comparisons. ${ }^{[10,11]}$

Our findings clearly demonstrate that SA HCWs from the Tshwane district of GP are at high risk of acquiring SARS-CoV-2 infection in comparison with the general population, but that these acquisition rates are in alignment with observations documented in several other countries. Compared with the initial report in August 2020, ${ }^{[3]}$ these findings are probably more reflective of the reality in SA public hospitals.

\section{Study limitations}

We acknowledge the following limitations: (i) poorly recorded data with missing variables for staff infections - for this reason, we excluded two hospitals for the in-depth analysis; (ii) the number of infections is likely to be underestimated, as all staff with infections may not have reported this to the OHSD; (iii) the small number of severe infections may have precluded meaningful statistical analysis; and (iv) we utilised the staff categories listed earlier for ease of use, but the level of exposure to patients may have varied within a group (e.g. a small proportion of medical doctors would have had minimal patient contact, and among nurses the extent of exposure would have varied depending on their duties) - however, we could not adjust for this, based on the available data captured by the OHSD and the fact that cross-discipline assistance and exposure were a reality during the surge.

\section{Conclusions}

This study highlights that hospital staff, including administrative staff, are at high risk for acquisition of SARS-CoV-2 infection during a surge, even with strict IPC practices and screening mechanisms in place. This increased risk may be attributed to increased workload, increased exposure of HCWs to patients, and cross-discipline work in unfamiliar environments, while the possibility of community acquisition should not be disregarded. At the time of writing, SA had experienced a second wave and was in the midst of a third wave. Patient numbers have increased with each subsequent wave globally, and did so in SA for the second wave. It would be interesting to review hospital staff acquisition rates for the subsequent surge periods, taking into account the higher patient numbers and the greater amount of patient sharing against the backdrop of the expected improvements in IPC adherence and vaccination uptake among the staff. It is evident that SARS-CoV-2 infections were not adequately recorded during the study period, at two of the hospitals in particular, and this needs to be reviewed and improved if necessary.

\section{Declaration. None.}

Acknowledgements. The OHSDs at SBAH, DGMAH and KPTH, and the human resources department at SBAH.

Author contributions. NM: protocol design, collection of data, seeking funding and writing of the article; MV: protocol design, collection of data, seeking funding and writing of article; CJvR: statistical analysis and writing of article; FP: protocol design and writing of article.

Funding. South African Society of Anaesthesiologists, Acacia Branch. Conflicts of interest. None.

Sim MR. The COVID-19 pandemic: Major risks to healthcare and other workers on the front line. Occup Environ Med 2020;77(5):281-282. https://doi.org/10.1136/oemed-2020-106567

2. Barello S, Palamenghi L, Graffigna G. Burnout and somatic symptoms among frontline healthcare professionals at the peak of the Italian COVID-19 pandemic. Psychiatry Res 2020;290:113129. https:// doi.org/10.1016/j.psychres.2020.113129

3. Department of Health, South Africa. Statement on health care workers that have acquired COVID-19. 13 August 2020. https://sacoronavirus.co.za/2020/08/13/minister-Zweli-mkhize-on-healthcareworkers-that-have-acquired-coronavirus-covid-19/ (accessed 14 June 2021).

4. Nguyen LH, Drew DA, Graham MS, et al. Risk of COVID-19 among front-line health-care workers and the general community: A prospective cohort study. Lancet Public Health 2020;5(9):e475-e483. https://doi.org/10.1016/S2468-2667(20)30164-X

5. Barrett ES, Horton DB, Roy J, et al. Prevalence of SARS-CoV-2 infection in previously undiagnosed health care workers in New Jersey, at the onset of the U.S. Covid-19 pandemic. BMC Infect Dis 2020;20(1):853. https://doi.org/10.1101/2020.04.20.20072470

6. Alajmi J, Jeremijenko AM, Abraham JC, et al. COVID-19 infection among healthcare workers in a national healthcare system: The Qatar experience. Int J Infect Dis 2020;100:386-389. https://doi. org/10.1016/j.ijid. 2020.09 .027

7. Piapan L, de Michieli P, Ronchese F, et al. COVID-19 outbreak in healthcare workers in hospitals in Trieste, North-East Italy. J Hosp Infect 2020;106(3):626-628. https://doi.org/10.1016/j.jhin.2020.08.012 Al Maskari Z, Al Blushi A, Khamis F, et al. Characteristics of healthcare workers infected with COVID-19: A cross-sectional observational study. Int J Infect Dis 2021;102:32-36. https://doi. COVID-19: A cross-sectional
org/10.1016/j.ijid.2020.10.009

9. Gómez-Ochoa SA, Franco OH, Rojas LZ, et al. COVID-19 in health-care workers: A living systematic review and meta-analysis of prevalence, risk factors, clinical characteristics, and outcomes. Am J Epidemiol 2020;190(1):161-175. https://doi.org/10.1093/aje/kwaal91

10. Statistics South Africa. Mid-year population estimates 2020. Statistical release P0302. Pretoria: Stats . Statistics South Africa. Mid-year population estimates 2020. Statistical release P0302. Pretoria: Stats
SA, 9 July 2020. http://www.statssa.gov.za/publications/P0302/P03022020.pdf (accessed 14 June 2021).

1. Marivate V, Arbi R, Combrink H, et al. Coronavirus disease (COVID-19) case data - South Africa. Marivate V, Arbi R, Combrink H, et al. Coronavirus disease (COVIL
Zenodo, 21 March 2020. https://doi.org/10.5281/zenodo.3819126

12. Black JRM, Bailey C, Przewrocka J, Dijkstra KK, Swanton C. COVID-19: The case for health-care Black JRM, Bailey C, Przewrocka J, Dijkstra KK, Swanton C. COVID-19: The case for health-care
worker screening to prevent hospital transmission. Lancet 2020;395(10234):1418-1420. https://doi, worker screening to prevent hospital
org $/ 10.1016 /$ S0140-6736(20)30917-X

13. Zou L, Ruan F, Huang M, et al. SARS-CoV-2 viral load in upper respiratory specimens of infected patients. N Engl J Med 2020;382(12):1177-1179. https://doi.org/10.1056/NEJMc2001737

14. Clemency BM, Varughese R, Scheafer DK, et al. Symptom criteria for COVID-19 testing of health care workers. Acad Emerg Med 2020;27(6):469-474. https://doi.org/10.1111/acem.14009

15. Yombi JC, de Greef J, Marsin AS, et al. Symptom-based screening for COVID-19 in health care workers: The importance of fever. J Hosp Infect 2020;105(3):428-429. https://doi.org/10.1016/j.jhin.2020.05.028 6. Zaina Al M, Ahlam Al B, Faryal K, et al. Characteristics of healthcare workers infected with COVID-19: A cross-sectional observational study. Int J Infect Dis 2021;102:32-36. http://doi. org/10.1016/j.ijid.2020.10.009

17. Zhou F, Yu T, Du R, et al. Clinical course and risk factors for mortality of adult inpatients with COVID-19 in Wuhan, China: A retrospective cohort study. Lancet 2020;395(10229):1054-1062. https://doi.org/10.1016/S0140-6736(20)30566-3

18. Jordan RE, Adab P, Cheng KK. Covid-19: Risk factors for severe disease and death. BMJ 2020;368:m1198. https://doi.org/10.1136/bmj.m1198

19. Zheng KI, Gao F, Wang X-B, et al. Obesity as a risk factor for greater severity of COVID-19 in patients with metabolic associated fatty liver disease. Metabolism 2020;108:154244. https://doi.org/10.1016/j. metabol.2020.154244

Accepted 16 September 2021 